\title{
DEVELOPING ETHICAL ENGINEERS WITH EMPATHY
}

\author{
Jennifer Howcroft, Kate Mercer, and Jennifer Boger \\ Systems Design Engineering, University of Waterloo \\ jenny.howcroft@uwaterloo.ca, kate.mercer@uwaterloo.ca, jboger@uwaterloo.ca
}

\begin{abstract}
Empathy-based skill development can help engineering students work towards professional expectations regarding ethical duties. However, there is a lack of explicit, holistic pedagogical approaches to empathy education in engineering. In BME161, a first-year biomedical engineering design course, students received explicit and implicit instruction focused on empathy and ethics. Students were also expected to use empathy-based tools and incorporate stakeholder perspectives in their design process in meaningful and explainable ways. While this approach was successful in incorporating empathybased education into a design course, a more holistic approach is needed throughout the program. Therefore, a high-level framework is presented based on four pillars of empathy development: communication, collaboration, decision-making, and values with a goal of achieving an interpersonal, user-centered, empathic culture of design in engineering students. Future work will focus on developing a more detailed and actionable framework.
\end{abstract}

Keywords: Empathy, Ethics, Education, Engineering design, Professional engineer, Case study

\section{INTRODUCTION}

Professional engineers are expected to conform to a code of ethics [1, p. 77], [2], [3]. These codes of ethics offer guidance and expectations regarding professional ethical expectations [4]. As such, ethics needs to be a core component of engineering education to ensure students have the skills and knowledge to face the ethical challenges and dilemmas inherent to their specific field of practice [4], [5]. Given the rapid pace of technological and scientific advancement in engineering fields, ethical training can be challenging and needs to engage students both intellectually and emotionally [5]. Developing empathybased skills has the potential to support emotional ethical training [6] and prepare students to meet the unknown ethical dilemmas they are sure to meet during their professional careers as they engage with other engineers (i.e., engineers within and outside their own discipline), collaborators (i.e., project and team members from nonengineering disciplines), and stakeholders (i.e., eventual users of designed systems). In other words, garnering empathy is fundamental to enabling ethical decision making.

Empathy as a concept in design is still all too often brushed aside in favour of a post-industrial mindset of mass-production of products that are designed for an 'average' user. In the 1940's, the United States Air Forces realized that designing cockpits and helmets for the 'average' user was one of the causes of a rise in aviation accidents: most pilots had body dimensions that deviated from the 'average' and experienced fit issues in these planes [7]. As designers of products for all of humanity, engineers must develop a deep understanding of the diversity of users, both physically and cognitively, and gain a true appreciation for the user's perspective which often differs from the engineer's perspective [8]-[11]. Using empathy to develop an understanding of stakeholder groups is a foundational skill for engineering students to learn - it has been linked to student's ability to consider stakeholder perspectives, integrate insights from various and often diverse stakeholders into design and decisionmaking processes, and, as demonstrated in the Air Force example, use this knowledge to improve design safety and user welfare [7], [10], [12]. By training engineers in methods of accessing and valuing stakeholder's (and others) perceptions, values, and lived experiences, engineers become professionally aware of, able to, and responsible for creating more responsible, inclusive, and appropriate products and systems throughout their careers.

For Professional Engineers Ontario (PEO), Engineers Canada (EC), the National Society of Professional Engineers (NSPE), and many other engineering societies, the most fundamental ethical duty is a duty to society and public welfare [1]-[3]. Empathy is needed to meet this fundamental ethical duty and can assist in some of the more specific ethical obligations placed on professional engineers which will be outlined in the subsequent paragraphs. Empathy has such strong professional ties in engineering that 'a professional way of being' was identified as one of three high-level dimensions in Walther et al.'s model of empathy [13].

While one could argue that all engineering ethical expectations would be better met by engineers with strong empathetic skills, there are some specific expectations where empathy has particular relevance. For PEO, some of the most relevant sections include, "1.i. It is the duty of a 
practitioner to... act at all times with fairness and loyalty to the practitioner's associates, employer, clients, subordinates and employees", and "1.ii. fidelity to public needs"; "2.ii. A practitioner shall...endeavour at all times to enhance the public regard for the practitioner's profession by extending the public knowledge thereof and discouraging untrue, unfair or exaggerated statements with respect to professional engineering"; " 6 . A practitioner must co-operate in working with other professionals engaged on a project", "7.i. A practitioner shall...act towards other practitioners with courtesy and good faith" [1, p. 77].

For EC, some of the most relevant sections include, "3. Act as faithful agents of their clients or employers, maintain confidentiality and avoid conflicts of interest, but, where such conflict arises, fully disclose the circumstances without delay to the employer or client", "5. Conduct themselves with integrity, equity, fairness, courtesy and good faith towards clients, colleagues and others, give credit where it is due, and accept, as well as give, honest and fair professional criticism", "8. Be aware of, and ensure that clients and employers are made aware of, societal and environmental consequences of actions or projects and endeavour to interpret engineering issues to the public in an objective and truthful manner", "9. Treat equitably and promote the equitable and dignified treatment of people in accordance with human rights legislation", and "10. Uphold and enhance the honour and dignity of the profession" [2].

For NSPE, some of the most relevant high-level canons include, "1. Hold paramount the safety, health, and welfare of the public", "4. Act for each employer or client as faithful agents or trustees.", and "6. Conduct themselves honorably, responsibly, ethically, and lawfully so as to enhance the honor, reputation, and usefulness of the profession" [3].

While empathy-based skill development has the potential to serve as a key component in training ethical engineering students to meet professional expectations, there is a lack of explicit, holistic pedagogical models focused on developing empathy in engineering students. This paper will focus on presenting the current state of empathy pedagogical models (Section 2), a case study of integrating empathy-development holistically into a firstyear design course (Section 3), and propose a high-level holistic pedagogical model of empathy and ethics in engineering education (Section 4).

\section{LITERATURE REVIEW}

\subsection{Empathy in the Engineering Context}

Engineering problems in the current world are often wicked problems: ill structured problems with no single right solution where appreciation for the unique and changing social and technological context is needed [14]. With wicked problems, a lack of empathy is often evident in weak final designs where the engineers have failed to fully appreciate the complexity of the problem, but in highquality final designs it can be challenging to identify the specific contribution of empathy within the final design [9].

Davis frames empathy as "the reactions of one individual to the observed experiences of another" [15]. However to fully explore the concept we must acknowledge that empathy at its core is the ability to see the world through other people's eyes [15]. Building on this and working under the definition of "empathy includes both affective experiences and cognitive processes that may be primed automatically or within the subconscious, and that may operate in isolation or concurrently, but which tend to have a cyclical relationship", engineering educators need to build empathy education in a holistic way that embeds itself throughout an engineering degree and promotes sustainable and just engineering practices [11], [12].

Incorporating empathetic design in engineering pedagogy hinges on providing students with meaningful opportunities to learn about, discuss, and experiment working with empathetic design practices. One of the challenges of accomplishing this is that there is a conceptual vagueness around concepts like empathy and care for practicing engineers, which aligns with the common perspective that empathy is not part of engineering culture [9]. Empathy is often given less importance compared to cost, liability, and technical acumen both by practicing engineers and in engineering education [9]. Parallel to this, engineers acknowledge that with empathy comes improved emotional intelligence, increased creativity, deeper understanding of problems and stakeholders, increased sustainability of designs, better communication (both with other engineers and various stakeholders), stronger innovation, improved effectiveness of work, and ultimately more professional success [6], [8], [9], [11], [13].

\subsection{Empathy \& Engineering Education}

Engineers must possess technical expertise, as well as other skills including collaboration, communication, empathy, and creativity [16]. Students need various types of knowledge to make ethical decisions, but they also need to develop the courage and confidence to ultimately see ethical decisions as a valuable aspect of their future as practicing engineers [17].

All engineers need to make ethical decisions, however biomedical engineers face unique ethical concerns compared to other engineering disciplines, where empathy is of particular benefit [17]. Navigating patient, medical records, treatments, animal testing, and collaboration with many different fields, including ones that have specific ethical guidelines unto themselves, some students can experience cognitive dissonance [17]. Further to this, in order to solve wicked biomedical problems, biomedical 
engineers need to deeply understand the people they are designing in addition to the broader health context. Finally, many engineers struggle to explain their decision making to non-engineers, which constitutes a significant barrier to the collaborations necessary for successful outcomes [18]. Therefore, engineers, particularly biomedical engineers, need to develop empathy skills focused on ethical decision making in design. As such, empathy-based education needs to be appropriately integrated into communication development, including the ability to explain this decisionmaking process.

The literature demonstrates that people are less likely to empathize with those who have different backgrounds to them, and it has been identified that this is a problem for engineering students [6], [18], [19]. Students can struggle when presented with a challenging empathy task where the culture and context is unfamiliar such as a problem space focused on the developing world [6]. Therefore, empathy education needs to be staged so that student empathy development is supported and not impeded.

An additional challenge with empathy education is that the human brain cannot analyze and empathize at the same time. As analytical training tends to dominate engineering education, this requires embracing a pedagogical shift that makes room for and values training in different cognitive ways of considering a problem space [10]. As challenging as it may be to marry the two in an engineering education program, empathy is crucial for decision making, communication, and strong design. However, empathy and care are not often explicitly represented in engineering education literature, and it has been identified that as students move through their degrees they become less interested in public welfare [10].

Disciplines outside engineering, such as medicine, do embed a more structured approach to teaching empathy in their curriculums [20]. While there are case-studies presenting empathy focused engineering education approaches in specific classes or connected to specific projects similar to this paper (e.g., [6], [10], [16]-[18]), there is a lack of an explicit, holistic pedagogical approach to empathy education in engineering. The remainder of this paper focuses on a case study demonstrating how empathy was embedded in a first-year undergraduate engineering design followed by a discussion of how to move toward the integration of empathy in engineering pedagogy in general.

\section{CASE STUDY: BME161}

BME161 - Introduction to Biomedical Design is a firstyear, mandatory design course with a typical enrollment of about 100 students. In this course, students engage in active learning of the iterative design process by solving an openended, real-world biomedical problem in teams of five to six students. In addition to this hands-on training, students attend lectures to build their foundational design knowledge and learn about broader topics related to professionalism and ethics. To effectively deliver these course components in an online manner, necessitated by the pandemic, the design project was reworked and staged around themed, weekly 'design sprints' listed in Table 1. Teams presented their progress in two design showcases that included team presentations and reports as well as individual design project contributions and related questions.

Table 1: Design Project Schedule.

\begin{tabular}{|c|c|}
\hline Week of Term & 'Design Sprint' Theme \\
\hline 1 & N/A - Course Welcome \\
\hline 2 & $\begin{array}{c}\text { Needs Assessment - Part 1: } \\
\text { Literature \& Standards Research }\end{array}$ \\
\hline 3 & $\begin{array}{c}\text { Needs Assessment - Part 2: Empathy } \\
\text { \& Ethics }\end{array}$ \\
\hline 4 & Synthesis \\
\hline 5 & Conceptual Design \\
\hline 6 & Design Showcase 1 - Reports \\
\hline 7 & Design Showcase 1 - Presentations \\
\hline 8 & Engineering Analysis \\
\hline 9 & Iterative Design \\
\hline 10 & Design Showcase 2 - Reports \\
\hline 11 & Design Showcase 2 - Presentations \\
\hline 12 & N/A-Course Wrap-Up \\
\hline
\end{tabular}

The design project for this online term focused on developing a way for individuals with spinal cord injury to measure their body weight at home as independently as possible.

\subsection{Empathy in BME161}

In addition to reworking the design project with a 'design sprint' framework suited to online learning, the instructor $(\mathrm{JH})$ made a strategic decision to take a holistic approach to empathy skill development where students would learn to practice empathy towards users, classmates, and instructors through both explicit and implicit instruction.

Empathy towards users occurred predominantly through explicit lectures materials and design project activities. In terms of lecture content, students were introduced to empathy at a rudimentary level and empathybuilding design techniques including personas and storyboards. In terms of the design project, students developed requirements and constraints based solely on published literature and standards in Week 2. Then in Week 3, they viewed three stakeholder interviews: two individuals with spinal cord injuries and one general practitioner specializing in caring for individuals with mobility challenges. After viewing these interviews, they were tasked with developing primary and secondary personas, and make at least two changes to the developed requirements and constraints that integrated insight gained from the stakeholder interviews. This additional insight had to be clearly articulated in the Design Showcase 1 
report. In Week 10, they developed a storyboard focused on user-product interactions depicting an individual with a spinal cord injury using the developed solution to determine their body weight. These tasks, in addition to developing design and empathy skills, work towards professional practices that meet engineering codes of ethics including, fidelity to public needs (PEO 1.ii; NSPE1), enhancing public regard for the profession (PEO, 2.ii), acting as faithful agents (EC3; NSPE4), and equitable and dignified treatment (EC9). Many of the students selfdirected toward empathetic, more inclusive design. For example, after viewing the stakeholder interviews, several teams broadened the identified user group to better meets the needs of the entire user population, even though it increased the challenge of the design project. Additionally, several teams incorporated user dignity as a key consideration after one stakeholder shared the experience of being weighed on an industrial scale in a workplace.

Developing empathy for classmates was more implicit. Students were encouraged to be mindful of the additional challenges and stressors of online learning and teamwork. This was indirectly assessed with a teamwork reflection assignment where students were asked about their team dynamic and how they could, at a team and individual level, improve this dynamic in future terms. Developing empathy for classmates has parallels with empathy for colleagues in the workplace which appears in several engineering code of ethics (PEO 1.i, 6, 7.i; EC5; NSPE6).

Developing empathy for instructors was explicit and implicit. In addition to similar conversations regarding the additional challenges and stressors of online learning, students were assessed on accountability and honest reporting of individual design project contributions which were reported and then further assessed with follow-up, individually targeted questions. This was aimed at developing professional engineering expectations regarding honesty and integrity in the workplace and appears in several engineering code of ethics (PEO 6, 7.i; EC5, 10).

\subsection{Ethics in BME161}

While one of the goals of the course was to develop a holistic approach to empathy skill development, ethical training was also an important part of BME161. Similar to empathy skill development, there were lectures focused on ethical topics including ethical guidelines (e.g., PEO Code of Ethics [1, p. 77], Ethical By Design - A Manifesto [21] Tech for Good [22]), ethics terminology (e.g., equity and equality), UN Sustainable Development Goals [23], and differences between engineering and healthcare codes of ethics.

In addition to this lecture-based content, students were expected to apply ethics into their design projects by developing an ethics-based requirement and a standardsbased requirement and explaining these requirements in the first Design Showcase. The ethics-based requirements focused on a variety of topics ranging from safe use to sustainable design choices to consideration of nonmobility impairments. The standards-based requirement focused on due diligence and ensuring that designs met minimum professional expectations in addition to providing students an opportunity to obtain and apply relevant information from standards to their design projects.

Finally, students were assessed on their ethical knowledge individually through two-targeted ethics questions focused on connecting ethical actions to professional engineering and explaining how the concept of equity applied to the design project.

\subsection{Key Observations from BME161}

Overall, the approach of empathy skill development combined with ethical instruction was effective in eliciting empathy in students towards the design project stakeholders (i.e., individuals with spinal cord injury). Students asked insightful questions regarding users' abilities, needs, desires, and existing supports as they worked towards developing meaningful solutions. For example, there were several questions regarding transfers focused on level of independence, associated technology, frequency of transfers, etc. Additionally, students incorporated insightful elements into their final designs that reflected empathy towards their users. For example, one team included a magnetic grabber device into their overall solution to assist users with accessing design elements that may fall to the floor. Another team integrated their device into a toilet seat so that it would be seamlessly integrated into the user's daily activities. While empathy is a challenging construct to measure in terms of appropriate application in the context of design, the students were more advanced (compared to previous years) at making empathetic design decisions compared to their peers in previous years.

In addition to the overall structure of the course discussed in 3.1 and 3.2, there were course elements that were critically important to the effectiveness of this approach: (1) design project topic, (2) instructor characteristics, and (3) the pandemic.

Firstly, selecting a meaningful, real-world design project where empathy is necessary to achieve a highquality design process and output was important. Realworld connection have been found to increase empathic experiences in engineering students [6]. The real-world nature of the problem meant that it was possible to work with actual stakeholders and provide the students with useful, targeted stakeholder interviews. The local nature of this problem (i.e., those interviewed were all in the Kitchener-Waterloo region) made the project more accessible to the students and made it easier to build empathy. Studies found that the selection of problem spaces associated with developing countries increased the 
challenge of and impeded empathy development for students [6], [19]. Additionally, it is important to select a problem space where developing empathy will be beneficial to the design process. While this means that the ultimate solution must involve a high degree of user interaction, groups that realise and design for this will are far more likely to have higher-quality results.

Secondly, instructor characteristics likely played a key role in the success of the holistic approach to empathy. The instructor needed to 'practice what they preach' [16], this meant demonstrating empathy towards the users associated with the design project and the students in the course. In addition to an empathetic mindset, the instructor also needed to be prepared to answer challenging user-focused questions to support student empathy development and applying empathy to the design project. This means the instructor must have strong familiarity with the users associated with the chosen problem space. For example, the course instructor for BME161 $(\mathrm{JH})$ is married to an individual with spinal cord injury and has been part of the spinal cord injury community as a caregiver for a decade. While this level of connection is not necessary, instructors need to be able to speak intelligently about the needs and concerns of the user population or have a stakeholder who is willing to assist with these types of questions.

Thirdly, the pandemic played an important role in increasing the success of holistically addressing empathy in BME161 as it placed additional challenges on students and instructor due to the unfamiliar nature of online learning. Given that everyone was experiencing these challenges together, it was appropriate and beneficial to discuss these challenges through the frame of empathy understanding that these challenges were impacting everyone in different ways and supporting each other throughout the term.

\section{TOWARDS A HOLISTIC PEDAGOGICAL MODEL OF EMPATHY \& ETHICS IN ENGINEERING EDUCATION}

While individual instructors can achieve success at integrating empathy and ethics into engineering courses in a meaningful, holistic way, the field of engineering education would benefit from a holistic pedagogical model of empathy and ethics in engineering education. This section presents a high-level framework for an empathybased pedagogical model with the intention of developing a more detailed pedagogical model in the future. This framework focuses on integrating empathy into design courses, similar to the case study presented in Section 3, with an overall goal of achieving an interpersonal, usercentered, empathic culture of design in engineering students. To achieve this goal, empathy development focuses on four pillars that are revisited throughout the undergraduate curriculum: communication, collaboration, decision-making, and values (see Fig. 1).

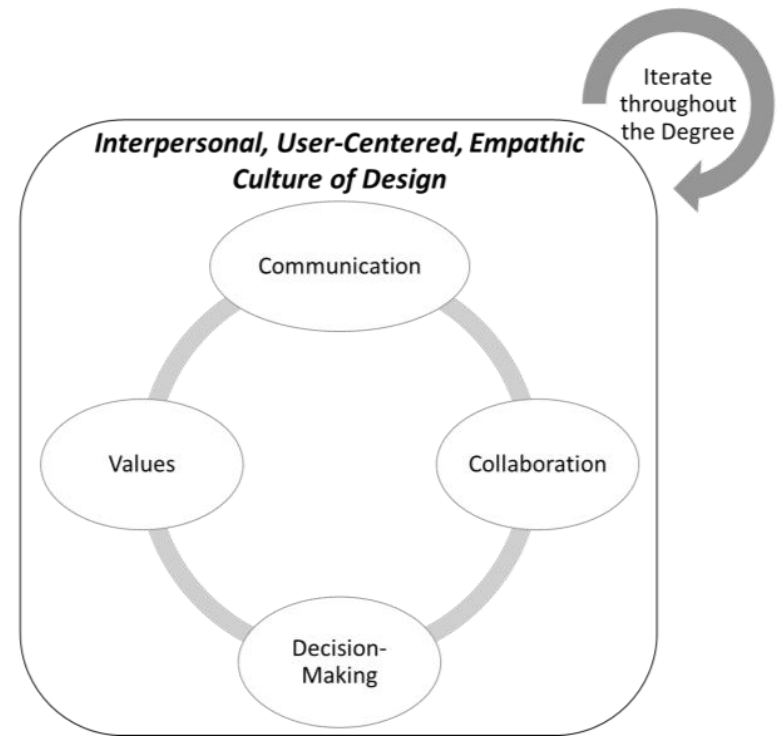

Figure 1. High-Level Framework for Empathy-based Engineering Education.

The first pillar is the incorporation of empathy into communication. Communication is critical throughout the design process: with stakeholders, clients, collaborators, and other engineers. In particular, communication is critical during user research where students need to appreciate and engage with stakeholders as complex humans with different interests, values, motivations, and ways of thinking instead of abstract or anonymous stakeholders [10]. In other words, students in biomedical engineering often characterize stakeholders as synonymous with their disability or diagnosis, rather than as individuals with needs and goals. Students need guidance and practice working with stakeholders to understand the role of empathy in these stakeholder interactions so that they can identify commonalities in stakeholder's needs and wants but also appreciate the diversity of stakeholder needs and wants so that appropriate solutions can be designed. Similarly, students need to learn the role of empathy in all social interactions, both personally and professionally, so they can better collaborate with peers, work collaboratively across different disciplines, interact and engage with stakeholders, understand the complexities of problems, and ultimately be more sustainable in their practice [12], [18].

One of the key goals of student development in the communication pillar is to view collaborators and stakeholders as equally important sources of knowledge as analytical investigations; that the viewpoints of others are as real and valid as their own. This supports the integration of meaningful multi-way, interpersonal communication between engineers, stakeholders, and collaborators throughout the design process that is necessary to achieve a high-quality final design. 
The second pillar is collaboration, which can only be successful if students have already integrated empathy into communication with their collaborators and stakeholders. To achieve interpersonal, user-centered, empathic design, engineering students must not only see collaborators and stakeholders as important sources of knowledge but also as important peers regarding ideas, regardless of differences in disciplines, education, etc. This has the potential to move engineering design from a 'paternalistic' model of decision-making to an 'interpersonal' model of decisionmaking, mirroring a similar transition in the healthcare field [24]. Participatory methods [25], such as those used in human factors, can be integrated into the engineering design process. This method encourages participation of stakeholders as active participants who inform the design process, increasing the potential of ultimate project success [25].

The third pillar is decision-making and builds on the first two pillars. Once engineering students have incorporated empathy into their communication and collaboration throughout the design process, empathic and ethical decision-making need to follow. Decision-making should focus on making design decisions that go beyond optimizing aspects such as cost, time, and other 'classic' engineering resources, to also respect the dignity of stakeholders, are appropriate for their contexts, and meet the most important ethical duty to society and public welfare [1]-[3].

Engineers often face complex decisions where it is not possible achieve high performance on all requirements and trade-offs are necessary to achieve an overall, workable solution. This becomes more complicated when trade-offs include both objective (e.g., cost, time, error rate, etc.) and subjective (e.g., perceived difficulty, relevance, dignity, etc.) measures. However, making good decisions regarding all these types of trade-offs is what is required of engineers practicing in real-world contexts. To navigate these tradeoffs, engineering students must consider all they have learned about their stakeholders needs and wants as well as engaging both their stakeholders and collaborators in meaningful conversations and the decision-making process itself. This collaborative decision-making process should be pervasive in engineering pedagogy; it should be modeled by instructors, demonstrated through casestudies, and practiced in design projects.

The final pillar is values - instilling empathy as an important, cornerstone value necessary for successful design. This last pillar is the most challenging, as it requires not only successfully instructing engineering students in empathic skill development but also changes in the culture of the engineering profession itself, which (in general) does not place high value on empathic skills [9].

Instructor modeling is an important for this pillar instructors must model behaviour that places emphasis on empathic skills and the integration of these skills and the gleaned insights into the design process and final design solutions. Instructors need to include empathic-focused feedback into student submissions; to reward students for engaging in incorporating empathy into their thinking process. As discussed in the Introduction, empathy is inherently connected with engineering code of ethics, which are a valued and integral component of professional engineering. Therefore, explicitly connecting these ideas in lectures can help students understand the importance of empathy in their development as future engineers and see empathy as integral to the profession.

External to the four pillars, the iterative component of the model is critical to ensuring that engineering students achieve a high-level of empathic skill. Engineering education focuses on analytic and technical courses. Given that the human brain cannot analyze and empathize at the same time [10], students benefit from multiple, focused opportunities to practice empathic skills on their own. This enables students to offset the analytic emphasis of engineering while also learning how to appropriately switch between analytic and empathic focuses throughout the design process.

Students need time to grow and develop their empathic skills. During their first year, students may need a more accessible empathic challenge, such as user populations with similar culture or regional backgrounds with whom it is easier to empathize. The empathic diversity can then be increased throughout the years to more challenging user populations like those with vastly different culture or regional backgrounds. This gradual approach will avoid student disengagement from empathy-focused tasks that can occur if the challenge is increased too quickly or too soon [6].

\section{CONCLUSION}

Empathy-based instruction can be holistically integrated into an engineering design course, as demonstrated in the BME161 case study, through both implicit and explicit instruction. Empathy is a complex concept, therefore to be effective, empathy must be taught throughout an engineering undergraduate degree in a holistic and increasingly complex manner. Doing so can reinforce to students and faculty alike that considering and complementing the contexts, needs, and values of the endusers and other stakeholders is an implicit core element of what it is to be a professional engineer.

This paper proposes a high-level framework of empathy-based instruction in design courses with a goal of developing an interpersonal, user-centered, empathic culture of design. This framework is based on four pillars of empathic development: communication, collaboration, decision-making, and values. In the future, this model needs to cultivated to include specific pedagogical techniques, case studies, design scenarios, and other instructional support tools that would support student development in each of the four pillars. 


\section{Acknowledgements}

We would like to thank the stakeholders who provided interviews in BME161 and the students who chose to explore outside their comfort zone as a result.

\section{References}

[1] "Section 77: Code of Ethics," Government of Ontario, R.R.O. 1990, Reg. 941, Jul. 2014. Accessed: Feb. 18, 2021. [Online]. Available: https://www.ontario.ca/laws/view.

[2] Engineers Canada, "Public Guideline on the code of ethics Engineers Canada." https://engineerscanada.ca/publications/public-guidelineon-the-code-of-ethics (accessed Feb. 18, 2021).

[3] National Society of Professional Engineers, "Code of Ethics | National Society of Professional Engineers." https://www.nspe.org/resources/ethics/code-ethics (accessed Feb. 18, 2021).

[4] J. Li and S. Fu, "A Systematic Approach to Engineering Ethics Education," Sci Eng Ethics, vol. 18, no. 2, pp. 339349, Jun. 2012, doi: 10.1007/s11948-010-9249-8.

[5] B. Newberry, "The dilemma of ethics in engineering education," Sci Eng Ethics, vol. 10, no. 2, pp. 343-351, Jun. 2004, doi: 10.1007/s11948-004-0030-8.

[6] M. Alsager Alzayed, C. McComb, J. Menold, J. Huff, and S. R. Miller, "Are you feeling me? An exploration of empathy development in engineering design education," Journal of Mechanical Design, pp. 1-57, Oct. 2020, doi: 10.1115/1.4048624.

[7] T. Rose, The End of Average - How We Succeed in a World that Values Sameness. Canada: Harper Collins Publishers Ltd., 2016.

[8] X. Tang, "From 'Empathic Design' to 'Empathic Engineering': Toward a Genealogy of Empathy in Engineering Education," Salt Lake City, Utah, 2018, p. 22414.

[9] J. Strobel, J. Hess, R. Pan, and C. A. W. Morris, "Empathy and care within engineering: qualitative perspectives from engineering faculty and practicing engineers," Engineering Studies, vol. 5, no. 2, pp. 137-159, Aug. 2013, doi: $10.1080 / 19378629.2013 .814136$.

[10] J. Walther, M. A. Brewer, N. W. Sochacka, and S. E. Miller, "Empathy and engineering formation," Journal of Engineering Education, vol. 109, no. 1, pp. 11-33, 2020, doi: https://doi.org/10.1002/jee.20301.

[11] J. L. Hess, J. Strobel, and R. Pan, "Voices from the workplace: practitioners' perspectives on the role of empathy and care within engineering: Engineering Studies:
Vol 8, No 3," Engineering Studies, vol. 8, no. 3, pp. 212$242,2016$.

[12] J. L. Hess and N. D. Fila, "The Development and Growth of Empathy Among Engineering Students," in ASEE, New Orleans, Los Angeles, 2016, p. 16281, Accessed: Feb. 18, 2021. [Online]. Available: https://scholarworks.iupui.edu/handle/1805/12192.

[13] J. Walther, S. E. Miller, and N. W. Sochacka, "A Model of Empathy in Engineering as a Core Skill, Practice Orientation, and Professional Way of Being," Journal of Engineering Education, vol. 106, no. 1, pp. 123-148, 2017, doi: https://doi.org/10.1002/jee.20159.

[14] J. Lönngren, T. Adawi, and M. Svanström, "Scaffolding strategies in a rubric-based intervention to promote engineering students' ability to address wicked problems," European Journal of Engineering Education, vol. 44, no. 12, pp. 196-221, Mar. 2019, doi: 10.1080/03043797.2017.1404010.

[15] M. H. Davis, "A multidimensional approach to individual difference in empathy," in JSAS Catalog of Selected Documents in Psychology, 1980, p. 85.

[16] J. Walther, S. E. Miller, and N. Kellam, "Exploring the role of empathy in engineering communication through a transdisciplinary dialogue," presented at the 119th ASEE Annual Conference and Exposition, 2012, Accessed: Feb. 18, 2021. [Online]. Available: https://asu.pure.elsevier.com/en/publications/exploring-therole-of-empathy-in-engineering-communication-throug- 2 .

[17] J. L. Hess, S. Miller, S. Higbee, G. A. Fore, and J. Wallace, "Empathy and ethical becoming in biomedical engineering education: a mixed methods study of an animal tissue harvesting laboratory," Australasian Journal of Engineering Education, vol. 0, no. 0, pp. 1-11, Jul. 2020, doi: $10.1080 / 22054952.2020 .1796045$

[18] G. Hoople and A. Choi-Fitzpatrick, "Engineering Empathy: A Multidisciplinary Approach Combining Engineering, Peace Studies, and Drones," Columbus, Ohio, 2017, p. 19372.

[19] C. Rasoal, H. Danielsson, and T. Jungert, "Empathy among students in engineering programmes," European Journal of Engineering Education, vol. 37, no. 5, pp. 427-435, Oct. 2012, doi: 10.1080/03043797.2012.708720.

[20] R. Pedersen, "Empathy development in medical education-a critical review," Med Teach, vol. 32, no. 7, pp. 593-600, 2010, doi: 10.3109/01421590903544702.

[21] M. Mulvenna, J. Boger, and R. Bond, "Ethical by Design: A Manifesto," in Proceedings of the European Conference on Cognitive Ergonomics 2017, New York, NY, USA, Sep. 2017, pp. 51-54, doi: 10.1145/3121283.3121300. 
[22] Canadian Innovation Space, "Tech for Good - A Declaration by the Canadian Tech Community." https://canadianinnovationspace.ca/tech-for-good/ (accessed Feb. 19, 2021).

[23] United Nations, "THE 17 GOALS | Sustainable Development." https://sdgs.un.org/goals (accessed Feb. 19, 2021).

[24] K. Mercer, "Communicating health information in primary care: a multidisciplinary exploration of patient, pharmacist, and physician decision-making: Thesis," University of Waterloo, Waterloo, Ontario, 2019.

[25] R. Burgess-Limerick, "Participatory ergonomics: Evidence and implementation lessons," Appl Ergon, vol. 68, pp. 289293, Apr. 2018, doi: 10.1016/j.apergo.2017.12.009. 